\title{
Manager les risques en projets : de la prise de conscience à la mise en confiance
}

Didier Gourc, Béatrice Vacher et Hervé Pingaud

\section{OpenEdition}

1 Journals

Édition électronique

URL : http://journals.openedition.org/communicationorganisation/2556

DOI : 10.4000/communicationorganisation.2556

ISSN : $1775-3546$

Éditeur

Presses universitaires de Bordeaux

Édition imprimée

Date de publication : 1 novembre 2001

ISSN : 1168-5549

Référence électronique

Didier Gourc, Béatrice Vacher et Hervé Pingaud, « Manager les risques en projets : de la prise de conscience à la mise en confiance », Communication et organisation [En ligne], 20 | 2001, mis en ligne le 27 mars 2012, consulté le 19 avril 2019. URL : http://journals.openedition.org/

communicationorganisation/2556 ; DOI : 10.4000/communicationorganisation.2556

Ce document a été généré automatiquement le 19 avril 2019

(c) Presses universitaires de Bordeaux 


\title{
Manager les risques en projets : de la prise de conscience à la mise en confiance
}

\author{
Didier Gourc, Béatrice Vacher et Hervé Pingaud
}

1 Dans le cadre d'un projet en entreprise, communiquer autour du risque n'est il pas un risque en soi ?

2 La plupart du temps, les objectifs de la décision de mise en place d'une démarche de management des risques sont tout à fait louables : mieux préparer les équipiers à une situation de crise, former aux conduites à tenir en cas de survenue, responsabiliser, etc. Toutefois, cet engagement peut avoir des effets aussi pervers qu'inattendus, comme par exemple affoler les membres des entreprises au point de les pétrifier, c'est-à-dire de les rendre inaptes à la moindre réaction.

3 À l'opposé, mener un projet d'envergure sans se lancer dans aucune démarche de management des risques peut aboutir à des résultats bien connus, notamment en matière de systèmes d'information : dépassement incontrôlable des budgets et des délais, conflits plus ou moins ouverts entre maîtrise d'œuvre, maîtrise d'ouvrage et utilisateurs, effets « usine à gaz » ${ }^{1}$ etc.

4 D'un côté, on communique sur le risque mais on peut bloquer des projets prometteurs, de l'autre côté, on fait aveuglément confiance au projet, au risque de n'obtenir aucun résultat.

5 Nous montrerons qu'une ignorance, involontaire ou feinte, des risques sur un projet est une source de danger, il est préférable qu'une prise de conscience intervienne le plus tôt possible. Il s'agit alors de sortir de l'impasse de l'inaction.

6 Nous proposons un agencement organisationnel (Girin, 1995) permettant de dépasser rapidement la seule prise de conscience pour passer à l'étape de mise en confiance seule capable de mobiliser les acteurs autour du projet, c'est-à-dire de déjouer l'inaction. L'agencement consiste en une combinaison d'outils particuliers que nous avons déjà eu 
l'occasion de mettre à l'épreuve (qu'il s'agisse de matériels ou de procédures) et une participation active des personnes selon une organisation de type «projet-risque » que nous décrirons et qui force à développer une vigilance mutuelle.

7 Nous argumenterons notre propos à travers quatre exemples significatifs : deux projets où une confiance aveugle est rapidement remise en cause et deux autres où une mise en confiance par la vigilance mutuelle suit immédiatement la conscience du risque. Ces cas sont tirés de travaux longs en entreprise menés par les auteurs : soit pour mettre en place (et à l'épreuve de la réalité) une méthode de management des risques sur des projets pharmaceutiques, soit pour étudier la mise en place de projets de systèmes d'information et les implications en matière d'utilisation.

Notre propos s'inscrit en grande partie dans la perspective des sciences du danger qui ont connu un essor important dans les deux dernières décennies. Liée à la volonté de maitriser des installations industrielles basées sur des technologies à risques (grandes installations chimiques, centrales nucléaires,...), l'approche systémique ${ }^{2}$ a fourni des éléments conceptuels pour analyser et gérer le risque industriel.

Une de ces sources de progrès est l'école des Cindyniques (Kervern, 1991). Les Cindyniques reposent sur les concepts de situation et de potentiel de danger (Kervern, 1999). Elles ont participé, et participent encore, notablement à la constitution de ce cadre d'approche des problèmes. La situation cindynique se réfère aux notions de champ d'étude (limites de temps, limites d'espace et réseaux d'acteurs) et de perception du danger. La perception est définie à travers cinq dimensions : épistémique, mnésique, téléologique, déontologique et axiologique. Les trois dernières dimensions cherchent à identifier le comportement des réseaux d'acteurs (respectivement en termes d'objectifs, de formes de régulation, de valeurs à respecter) et traversent explicitement le champ de la communication autour du risque industriel (Wybo, 1998). La communication du risque est donc incluse dans l'analyse du risque pour cette école de pensée, elle en est partie prenante.

La progression de ces travaux dépasse le cadre strict du risque industriel d'origine technique pour s'appliquer à l'évolution récente des techniques de management d'entreprise. Dans une communication présentée lors du colloque « Risque et société » en 1998, P. SONIGO (Sonigo, 1998) exprime les manques perçus par les industriels en matière de gestion des risques. Selon lui, dans un contexte où : l'occurrence d'un événement accidentel peut affecter durablement la gestion financière, l'image, les relations de partenariats et les motivations des employés, la non maitrise des aléas est devenue inacceptable pour la hiérarchie, et souvent par la loi, l'entreprise aura intérêt à confier à des «Risk Manager» le soin de maîtriser les différentes formes de risque de façon globale.

11 Nous avons donc à notre disposition d'une part, une base de savoir et de savoir faire qui s'est construite autour du risque industriel induit par la technologie et s'en est émancipé, et d'autre part, des industriels qui appellent de leurs vœux la mise en place de nouvelles organisations aptes à généraliser la démarche et à prendre en charge le risque sous ses différentes formes.

La gestion par projets est une forme d'organisation de plus en plus fréquemment adoptée par les entreprises. Nous avons donc un contexte industriel où il faut réaliser un ensemble d'activités dont la performance finale est classiquement évaluée par des indicateurs de coût, de délai et de qualité. La notion de management intégré tend à ouvrir 
ce spectre d'indicateurs à d'autres performances exprimées vis-à-vis de l'environnement et de la sécurité. C'est le fameux QHSE $^{3}$ dont un des enjeux est de conforter l'image d'une entreprise responsable (Fumey, 2001). Ce terrain du projet forme donc pour nous un microcosme de taille réduite propice à l'observation et à l'analyse de la communication autour du risque. Nous avons volontairement restreint le périmètre de l'étude à la communication à l'intérieur du projet. Même si les échanges internes à l'équipe projet sont forcément influencés par l'image que l'environnement a du projet, nous avons cherché à sélectionner des illustrations où cette influence est négligeable en première approximation.

\section{Prise de conscience du risque ou confiance aveugle dans le projet?}

Les multiples définitions et concepts véhiculés par les termes risques et dangers (principalement dans les acceptions latines et francophones) induisent, dans l'esprit des individus, une connotation négative voire hostile (March, 1989). Nous définissons le risque projet comme «la probabilité que le projet ne s'exécute pas conformément à une référence préalablement établie » (Giard 1991). Plus généralement, nous introduisons la notion d'événement, qu'il soit redouté, indésirable ou inespéré, afin de préciser la nature de l'écart par rapport à une situation de référence. Cela nous permet, entre autres de faire apparaître un concept important qui est celui du «risque positif» ou plus clairement de l'opportunité à saisir.

Mais la mise en place d'une démarche de maîtrise des risques peut avoir pour effet d'engendrer des attitudes suspicieuses auprès des personnes concernées. Les réticences des individus peuvent être exacerbées d'autant plus qu'ils n'y auront pas été préparés culturellement et techniquement. Par exemple, cette unité de production pharmaceutique qui dans le cadre d'un changement technologique sur une de ses lignes de production décide d'engager une démarche d'analyse des risques. Cette action, la première vécue par les opérateurs et pilotes de la ligne, a fait ressortir des risques sérieux (pour les opérateurs, pour la ligne) induits par la mise en œuvre de cette nouvelle technologie. La conséquence directe a été, non pas un refus d'appliquer cette nouvelle technologie mais un frein de la part des opérateurs, lors de la réalisation des premiers essais. Autre exemple, le directeur de cette entreprise de travaux publics, appelée à construire ses propres nouveaux bâtiments et à y emménager, informe son personnel que ces opérations ont lieu au même moment que celles du concurrent le plus important. Le directeur souhaitait motiver ses employés par un défi à relever mais ces derniers ont pris peur, relativisant leurs faibles moyens de travail par rapport à ceux du concurrent. Ils pensaient ne jamais réussir et s'opposaient à toute initiative pour organiser les équipes de façon à gagner en efficacité pour relever le défi : « Ca ne sert à rien, juste à prouver qu'on est mauvais! », tel était leur refrain.

Dans ces cas précis, la réaction de l'équipe traduit-elle une crainte instinctive du danger (par exemple de la gravité de l'impact du risque), de son caractère exceptionnel et aléatoire (c'est-à-dire la probabilité que survienne l'événement redouté) et donc de sa difficile maîtrise ou bien était-elle tout simplement le résultat d'une réflexion conduisant à des actions mieux maîtrisées pour augmenter le niveau de sécurité ou les chances de réussiri ${ }^{4}$ ? 
16 La prise de conscience du risque par les acteurs du projet ainsi que ses éventuelles conséquences ne sont-elles pas plus néfastes qu'une politique permettant de laisser les opérateurs dans une situation de confiance?

Nous pensons que cette confiance est aveugle, dangereusement aveugle.

Dans une organisation favorisant ce type de confiance par rapport à une prise de conscience du risque, le management peut être perçu comme chaotique avec des décisions en forme de marche d'escalier. Nous entendons par là, un management qui, confronté à des perturbations inattendues, doit prendre des décisions à chaud pouvant devenir contradictoires avec des orientations antérieures. La prise de décision est souvent rare dans ce contexte et fréquemment source de démotivation pour les équipiers.

Ainsi, l'exemple d'un projet de R \& D (Gourc, 2000 ; Bougaret, 2000) réputé sans problème et présenté comme tel par le chef de projet lors d'un comité de pilotage et qui, sous l'influence d'un effet d'annonce d'un équipier, sombre dans le chaos. Il suffit de très peu de choses en effet: l'équipier apporte une information, généralement catastrophique pour le projet et non divulguée précédemment au chef de projet, lors d'une réunion plénière en présence de l'ensemble des directeurs. Avec quelques talents scéniques, le calme devient turbulence, les priorités d'actions sont totalement modifiées, toute l'organisation s'écroule, les missions assignées à chacun s'en trouvent bouleversées.

20 Au-delà de cet exemple, l'absence de circuits d'informations clairement établis et de règles de communication formalisées confrontée à quelques "grandes gueules " est à l'origine de réunions projet qui voient ainsi passer un courant d'air alarmant, voire alarmiste, sur toute l'équipe avec la naissance d'un fort sentiment de démotivation. La conséquence immédiate est l'impossibilité de maintenir l'objectif initial de la réunion sans pour autant permettre la résolution du danger présenté. En effet, les participants à la réunion sont surpris devant l'inattendu et donc ne peuvent se concentrer sur la résolution du problème (phase de focalisation sur l'inattendu), ils n'ont pas suffisamment de données pour préparer et identifier des actions de traitement (phase de désorganisation devant l'inattendu), ils n'ont pas le temps matériel pendant la réunion d'organiser leur réflexion (phase de stress devant l'urgence) et ce d'autant que cette réflexion nécessite fréquemment la consultation d'experts ne participant pas à la réunion. À plus long terme, le projet peut souffrir d'une image plus tragique en véhiculant l'archétype du projet à risque, quelque soit la réalité.

21 A contrario, une trop grande formalisation et un manque de confiance dans le personnel peuvent présenter un risque similaire: dans le cas de l'Assurance Qualité dans le nucléaire par exemple, la multiplication des procédures et l'automatisation des tâches est si importante qu'elle cache aux dirigeants, et par là au public, la réalité des actions menées. On fait une confiance aveugle dans le système formel et informatique alors que ceux qui assurent la Qualité au quotidien n'apparaissent même pas dans l'organigramme officiel. Confrontée à la grève du zèle, c'est-à-dire au refus par le personnel d'en faire plus que ce qui est inscrit dans les procédures, quels risques cette entreprise prend elle? (Vacher, 2001)

22 Tout projet présente des risques, plus ou moins importants et demandant des actions plus ou moins rapides, fortes, contraignantes, etc. Il s'agit de les évaluer et de les prendre en compte de la façon la plus rassurante pour les opérateurs. Pour assurer ce travail, nous examinerons comment l'organisation peut être mise à contribution pour maitriser des risques pendant le pilotage de projets. 


\section{Vers la mise en confiance : une question d'organisation}

23 Le management des risques en projet consiste traditionnellement à prendre en compte les événements potentiels, pour réduire, sinon supprimer, leur probabilité de survenue et/ou leurs conséquences (AFITEP/AFNOR, 1996; Marmuse, 1989). En outre, J. Laboulet, (Laboulet, 2001) définit en première approche le management des risques comme permettant «de conduire les responsables de projet à des actions qui, prenant en compte les risques potentiels, viseront soit à les éviter en trouvant, par exemple, des solutions alternatives, soit à les réduire, soit à en réduire les conséquences pour les rendre acceptables ». Nous proposons de compléter cette liste d'objectifs :

24 - aider à l'obtention d'une meilleure connaissance de la situation du projet et de son niveau d'exposition au risque par la mise à disposition d'indicateurs complémentaires pertinents,

25 - améliorer l'anticipation et la réactivité des équipes projet à la survenue des événements redoutés, ou opportuns,

26 - participer à une meilleure définition de la cible du projet au lancement de celui-ci, mais également tout au long du projet, par une réactualisation périodique en fonction du degré de connaissance croissant du projet. Différents auteurs (DGA/AQ924; Courtot, 1999) ont étudié et/ou développé des démarches de management des risques et proposé des classifications en fonction des types d'outils ou méthodologies utilisés (quantitatifs, qualitatifs, hybrides, simulatoires, etc.) et des phases proposées. Dans la plupart des démarches identifiées, on retrouve un processus, itératif et cyclique, composé de cinq phases qui est mené tout au long du projet :

27 - l'identification des événements conduisant à la constitution d'une liste aussi exhaustive que possible des événements et des types de conséquences (impacts) que leur survenue pourrait entraîner,

28 - l'évaluation et la hiérarchisation des risques pour chaque projet, cette phase permet de quantifier chacun des risques (probabilité de survenue, gravité des impacts), afin de pouvoir trier la liste des risques par ordre d'importance (par ex. selon le critère de criticité qui correspond au produit de la probabilité de survenue et de la gravité). On obtiendra ainsi la liste des risques «majeurs » à considérer lors de ce processus. Cette démarche doit être menée régulièrement afin de tenir compte de l'évolution du projet (des connaissances sur le projet) mais également du cycle de vie intrinsèque des risques,

29 - la prise en compte de ces risques, conduisant à identifier et décider des actions à mener pour traiter les risques majeurs,

30 - le suivi des actions, permettant de surveiller la mise en œuvre des actions décidées et d'identifier leurs effets,

31 - la capitalisation et le retour d'expérience. Cette dernière phase doit comporter une tâche de constitution du retour d'expérience et de réalisation d'un dossier de suivi.

32 En complément à ces approches, la première nécessité dans une organisation projet dédiée à la maîtrise des risques est de définir clairement les rôles, missions et responsabilités de chacun. En première intention cela s'applique à la démarche classique de gestion de projet qui conduit à identifier le responsable du processus de maîtrise des 
risques présentés précédemment. En deuxième intention, cela s'applique aux actions de maîtrise des risques qui seront placées sous la responsabilité d'un acteur désigné.

Dans la pratique cela peut prendre par exemple la forme d'une matrice des responsabilités des acteurs du projet. Ainsi chaque lot de travaux du projet, chaque phase du processus de maîtrise des risques, chaque action à mener est sous la responsabilité d'un équipier, désigné par le chef de projet en relation avec les responsables de ressources. Les responsables sont clairement identifiés et connus de tous. Chaque responsable est lui-même informé de ses responsabilités et devoirs et les accepte.

Cette organisation se manifeste également par une précision des actions à mener et menées et de leurs objectifs : chaque réunion fait l'objet d'un ordre du jour, chacun formalise et accepte les objectifs de chaque réunion, l'animateur assure le respect des objectifs, chaque nature de problèmes est prise en charge par un comité spécifique, etc. Les comités peuvent être de trois sortes :

35 1) stratégique : réunion multi-projets (préoccupations multi-projets, prise en compte des risques financiers pour l'entreprise, priorisation des projets, affectation des ressources, cohérence des actions, etc.),

36 2) pilotage projet: réunion multi-métiers et mono-projet (problèmes d'organisation interne au projet, analyse des risques projet, marketing, relations inter-métiers, avancement technique calendaire et budgétaire du projet),

37 3) technique métier : réunion mono-métier (problèmes spécifiques à chacun des métiers). Cette organisation, doublée avec des contacts fréquents entre le chef de projet et chacun des métiers, permet également de favoriser l'anticipation des dangers par la communication au chef de projet des soupçons ou des précurseurs identifiés. La valorisation de ce type de comportement est d'ailleurs cruciale.

Mais quelle est cette organisation quasi-magique qu'il faut mettre en place pour tout savoir sans soupçonner personne? Quel est ce héros, le chef ou directeur de projet, qui peut définir clairement les rôles et mener le projet en toute sérénité quels qu'en soient les risques?

Dans les situations nécessitant une grande fiabilité, K. Weick et K. Roberts (1993) ont dégagé trois principes permettant le développement de ce type d'organisation menant à la vigilance mutuelle et par-là, à l'intelligence collective et à la performance organisationnelle : la contribution de chacun au système collectif, la représentation de ce système comme un ensemble d'actions liées et la subordination des actions individuelles au système. En d'autres termes, soumis à des contraintes fortes, les acteurs construisent leurs actions (contribution) en considérant que le système dans lequel elles s'insèrent doit être un ensemble d'actions connectées les unes aux autres (représentation) et que les leurs doivent être en étroite relation avec ce système (subordination des actions). Cette attention de chacun sur les actions des uns et des autres, ou vigilance mutuelle, se manifeste dans l'interdépendance: chaque action est construite pour dépendre des autres, chaque acteur accepte de dépendre des autres. Cette interdépendance n'est pas liée à l'ordre hiérarchique mais à la distance plus ou moins grande du cœur du projet. Chaque nouvel entrant est l'occasion de reconstruire la vigilance interne du système en racontant les histoires.

Pour assurer ce type de vigilance, les qualités d'écoute, de concentration et de motivation du chef de projet sont en effet primordiales (Midler, 1993) mais l'équipe n'est pas forcément exceptionnelle. Pour mobiliser la majorité des personnes sur un projet, il suffit 
d'un ou de deux acteurs bien placés (Akrich \& al, 1988 ; Vacher, 1997) : la construction d'un réseau, c'est-à-dire la façon dont les enjeux du projet sont traduits selon les types d'acteurs, est plus importante que le choix de techniques et de personnel les plus performants. Construire ce réseau, c'est pour ce noyau dur du projet un travail quotidien pour enrôler les «neutres", désarmer les " ennemis » et stimuler sans cesse les " amis ». On peut parler de manipulation au sens où la majorité des acteurs (les neutres) ne souhaitent pas s'engager dans le projet à risque; dans ce cas, précisons, comme le montrent très clairement J.-L. Beauvois et X. Joule (1987), que la manipulation la plus réussie est celle à l'issue de laquelle l'acteur décide lui-même de s'engager dans l'action. Dans ce cas, il sera le meilleur défenseur du projet (il ne peut pas faire machine arrière), il rentre définitivement dans la catégorie des amis.

Le principal ennemi dans la prise en compte opérationnelle des risques est l'habitude qu'il faut déjouer, celle de confondre la recherche des causes, des coupables et des responsables. La formation est par ailleurs un excellent stimulant et la traduction du risque dans le langage métier du personnel un très bon moyen d'enrôler ce personnel qui se sent alors concerné.

Pour développer ces différents points, nous proposons une double approche: par les outils et les symboles (dont les procédures) d'abord, par les hommes et l'organisation ensuite.

\section{Des outils concrets et symboliques}

Le premier outil participatif très efficace que nous proposons est le retour d'expérience basé sur les éléments factuels (Goure, 1999; Chapman, 1998). Il s'opère par entretiens, brainstorming, analyse de documents projet, approches type Delphi. On s'intéresse à la recherche des aléas vécus lors d'expériences antérieures en s'attachant à considérer uniquement les faits : quel aléa? Quelles causes techniques? Quels éléments précurseurs de la survenue de l'aléa ? Quand ou à quel moment dans le projet? Quelles conséquences ? Quelles actions menées? Quel résultat ou effet? Il s'agit d'éviter les débats de responsabilité, plus révélateurs de conflits interpersonnels que susceptibles d'aider à la prise en compte des risques. Ce processus essentiellement basé sur la mémoire des individus et leur interprétation des faits nécessite d'appuyer la démarche sur la méthode Delphi, méthode d'obtention de consensus, qui permet de s'affranchir des avis et opinions divergents en dépassionnant les débats.

Ce diagnostic, réalisé dans un climat de confiance, permettra de construire une démarche d'anticipation basée sur l'identification des aléas par les acteurs opérationnels. Ce sont en effet ces derniers qui peuvent le mieux renseigner sur la survenue probable d'aléas par la détection d'éléments précurseurs. Pour un bon fonctionnement de ce système, il faut qu'ils aient l'assurance que la transmission des soupçons ou des aléas avérés aux décideurs ne les rende pas responsables de la survenue d'un événement indépendant de leur bonne volonté.

Anticiper seulement les aléas ne suffit pas, cela reviendrait juste à préparer l'effet d'annonce qui peut être aussi dangereuse que l'ignorance des risques potentiels du projet. La communication des actions de traitement du risque doit être simultanée à celle du risque lui-même et les actions être menées rapidement; elles auront alors le mérite de rassurer les équipes, de mettre en confiance le personnel. 

risques a proposé les procédures de décision ou d'aide à la décision à suivre face à la survenue des aléas potentiels lors des essais d'exploitation du nouveau procédé ; ainsi chacun savait exactement ce qu'il avait à faire.

47 L'exemple de l'entreprise de travaux public est également significatif à cet égard. Un logiciel d'aide au déménagement a été développé pour répondre aux besoins des différents acteurs opérationnels du projet (standardistes, services généraux, services du personnel, informaticiens et téléphonistes, etc.). Ces acteurs ont été souvent consultés, réunis, formés, écoutés pour modifier le logiciel, soutenus dans leur utilisation quotidienne pour "bricoler» le logiciel à leur convenance et une communication régulière des avancées des travaux était faite au comité de direction de l'entreprise. Le président intervenait lui-même en cas de problème sur le terrain. La mobilisation constante de l'équipe de direction et l'intérêt porté systématiquement aux travaux les plus prosaïques a non seulement rassuré les ouvriers sur la capacité de l'entreprise mais les a motivés pour s'impliquer eux-mêmes en permanence et être force de proposition. Un chef des opérations, un téléphoniste aux idées neuves et un développeur informatique ont permis de mobiliser une équipe de trente personnes pour assurer le déménagement de trois mille personnes.

48 Communiquer sur le risque permet de créer une urgence. Pour que cette urgence devienne mobilisatrice et permette de créer de l'engagement et de la vigilance mutuelle (à tous les niveaux hiérarchiques comme l'illustrent les exemples), il est nécessaire d'agir rapidement en conséquence. Il s'agit donc de travailler en situation d'anticipation plutôt qu'en situation de crise, ce qui permet d'élargir l'ensemble des actions de traitement des risques que l'on peut envisager. On peut alors considérer des actions :

49 - préventives, c'est-à-dire prise avant la survenue de l'aléa et dont le mécanisme agit à tout moment. On trouvera notamment les actions visant à diminuer la probabilité de survenue par une mesure d'évitement du risque (ex: pose de ralentisseurs avant un virage dangereux) et les actions de réduction des effets induits si l'aléa survenait (ex: pose de barrières de protection),

50 - correctives, visant à corriger l'ampleur des effets induits après la survenue de l'aléa (ex : appel d'un service de soin),

51 - curatives, permettant de remédier définitivement au risque (ex : modification du tracé de la route).

En situation de crise, seules les actions correctives peuvent ensuite être développées. Mais une action corrective définie au moment de la crise peut s'avérer pire que ne rien faire. Les catastrophes vécues récemment sont là pour le rappeler, les conséquences, que tout le monde connaît aujourd'hui, ne sont en fait que le résultat d'un enchaînement de décisions prises à chaud et d'actes qui n'ont fait qu'amplifier les effets d'un événement mineur à l'origine ${ }^{5}$.

53 Bien évidemment, ce type d'outils à développer pour maitriser les risques (les procédures du groupe pharmaceutique ou le logiciel d'aide au déménagement par exemple) a un coût. Dans le fonctionnement idéal, le noyau dur du projet doit comparer les efforts à consentir aux bénéfices à en retirer. Il s'agit d'évaluer le ratio bénéfice/coûts. Ce ratio est utilisé au cours du processus de maitrise des risques et notamment lors de la phase de quantification des risques. Il permet de hiérarchiser les risques par degré d'importance en fonction (1) de la criticité du risque et (2) de la « rentabilité » des actions de traitement 
envisagées et ainsi de concentrer les efforts sur les risques qui méritent d'être pris en compte dans cette démarche. Ce critère est également utilisé pour lever l'indécision entre différentes actions possibles pour traiter un risque. Dans les faits, l'utilisation de ce critère nécessite tout de même une organisation familière avec la culture risque et doté d'un retour d'expérience suffisamment complet afin de pouvoir mener les estimations nécessaires pour déterminer ce ratio. Ainsi bon nombre d'entreprises commencent par développer la capacité d'action des individus en situation risquée avant de mettre en place des démarches plus lourdes.

\section{Les personnes : l'organisation « projet-risque »}

d'une fonction dédiée à la maîtrise des risques peut s'avérer nécessaire: «Risk Management ». La mission de cette structure, et de son responsable est de coordonner la mise en œuvre opérationnelle de la démarche de maîtrise des risques auprès et avec les équipiers (harmonisation des procédures, mise à disposition des outils adaptés, aide aux équipiers, etc.). La mission peut différer en fonction du contexte et du type de projet concerné. Ainsi, sur des projets de grande taille (effectif important, budgets élevés, enjeux forts, etc.) ou dans des organisations multi-projets (partage de ressources communes: humaines, machines, etc.), le «Risk manager " doit veiller à ce que les démarches appliquées soient adaptées à chaque projet (ou sous-projet) tout en maintenant une harmonisation des pratiques afin d'éviter un rejet de la part des acteurs concernés qui pourraient voir des pratiques diamétralement opposées suivant le projet sur lequel ils travaillent.

fois dans les faits, cette fonction bien que nécessaire et compatible avec la distribution de la vigilance auprès de tous les acteurs du projet est assumée par le chef de projet qui cumule ainsi les fonctions de " project manager » et de « project leader ».

\section{Conclusion}

La prise de conscience du risque sous ses différentes formes est donc un stade d'évolution des organisations qui est aujourd'hui atteint, et qui pose clairement la question de la 
communication au sein de l'équipe. En effet, la communication sur le risque présente un risque sur le facteur humain, qui se traduira par une démotivation ou une perte de performance si un certain nombre de mesures ne sont pas mises en œuvre au niveau de l'organisation. Pour agir, c'est-à-dire manager les risques en projet, il y a des solutions au niveau des outils matériels et symboliques et au niveau des hommes: rechercher des aléas sans chercher de coupable, communiquer les actions en même temps que communiquer sur le risque, mettre en place les outils de gestion correspondant assez vite (procédures, évaluation des enjeux-risques, logiciels), choisir une équipe pluricompétente et de sensibilités complémentaires au risque. Le rôle principal du noyau dur du projet est de faire en sorte que l'ensemble des acteurs s'engage dans la maîtrise des risques au sens de l'attention sur les actions mutuelles.

Nous sommes en train de prolonger ces travaux par une étude spécifique des projets de système d'information. C'est un contexte où l'expression du besoin est réputée être une étape difficile parce que l'objet de l'étude est de nature immatérielle. Au delà des solutions techniques préconisées pour la gestion des projets de système d'information, on sent qu'il existe des freins dans la mise en œuvre liés aux modes de communication entre le maitre d'ouvrage, le maître d'œuvre et les cabinets de consultant. Lever ces barrières implique une démarche de maîtrise des risques. La mise en scène des projets de système d'information va donc constituer, pour les auteurs, un terrain d'application privilégié où on ne va plus seulement examiner la communication interne, mais également les interactions avec les acteurs situés dans l'environnement du projet, et donc travailler les communications en fonction des rôles attribués à ces acteurs.

\section{BIBLIOGRAPHIE}

AFITEP/AFNOR, Dictionnaire de management de projet, 1990 AKRICH, M., CALLON, M., LATOUR, B., A quoi tient le succès des innovations. Premier épisode : L'art de l'intéressement et Deuxième épisode : L'art de choisir les bons porte-parole. Gérer et Comprendre, juin et Septembre, pp. 4-17, 1988

BEAUVOIS, J. L., JOULE, X., Petit traité de manipulation à l'usage des honnêtes gens. Presses Universitaires de Grenoble, 1987 BOUGARET, S., GOURC, D., Risques et ressources humaines en situation projet. In Ressources humaines et projet : Congrès de Management de projet, $16^{\mathrm{e}}$ congrès, 7-8 novembre 2000, Paris, 2000

BRINER, W., GEDDES, M., HASTINGS, C, Le manager de projet : un leader. Edition Afnor Gestion, 1993

CHAPMAN, R.J., The effectiveness of working group risk identification and assessment techniques. International Journal of Project Management, Vol 16, nº 6, 1998, p 333-343

COURTOT, H., Les risques liés à la gestion des acteurs d'un projet. Rapport interne IAE Paris, 1998

COURTOT, H., La gestion des risques dans les projets. Economica, 1999 DGA/AQ924, Manuel du management des risques dans les programmes d'armement. Direction Générale à l'Armement, Edition juin 1995 FUMEY, M., Méthode d'Evaluation des Risques Agrégés : application au choix des investissements de renouvellement d'installations. Thèse de doctorat, centre de Génie Industriel de 
l'École des Mines d'Albi, 2001 GIARD, V., Pilotage de projets et d'entreprise. Economica, 1991. GIRIN, J., Le langage et la compétence des agencements organisationnels. Connexions, Éditions érès, $\mathrm{n}^{\circ} 65$, pp. 121-141, 1995

GOURC, D., BOUGARET, S., Le management des risques appliqué au management de projets et de portefeuille de projets. Revue Travail et Méthodes, juillet 2000, n 552, pp 35-42

GOURC, D., BOUGARET, S., LACOSTE, G., Difficultés liées à la collecte et capitalisation des informations relatives aux risques techniques des projets innovants dans une structure matricielle. In L'intégration des ressources humaines et des technologies : le défi : texte du $3^{\text {ème }}$ Congrès International de Génie Industriel, 26-28 mai 1999, Montréal, 1999

GROSSET-GRANGE, Le chef de projet pouvoirs, responsabilité dans la gestion d'un projet. Etudes FIEV, 1990

KERVERN, G. Y, RUBISE, P., L'archipel du danger. ED. Economica, 1991 KERVERN, G. Y, Le point sur les cindyniques, Ecole d'été sur la gestion scientifique du risque. Albi, France, 6-10 septembre 1999 LABOULET, J., Les risques, chapitre de l'ouvrage Management de projet, Editions WEKA, Méthodes et outils tome I, 2001

MARCH, J.G., SHAPIRA, Z., Les managers face au risque. In Décisions et organisations, traduit de Decisions and Organisations par M. Waquet, Les éditions d'organisation, pp 109-130, 1989

MARMUSE, C, MONTAIGNE, X., Management du risque. Vuibert Entreprise, 1989

MIDLER, C, L'auto qui n'existait pas. InterÉditions, Paris, 215 p., 1993 SONIGO, P., Autour des notions de danger et de risques. Le point de vue de l'industriel. In Risque et Société, pp 63-68, Editions Nucléon, 1999 VACHER, B., La gestion de l'information en entreprise. Enquête sur l'oubli, l'étourderie, la ruse et le bricolage organisés. ADBS Editions, 231 p., 1997 VACHER, B., Faire ou prouver ? L'écrit de l'Assurance Qualité. In Le langage dans les organisations - Une nouvelle donne, textes réunis par S. Pène, A. Borzeix, B. Fraenkel, Collection Langage \& travail, Paris : L'Harmattan, pp. 115-130, 2001 WEICK, K. E., ROBERTS, K. H., Collective mind in organisations : Heedful interrelating on flight decks. Administrative Science Quaterly, Vol 38, n 3, septembre, pp. 357-381, 1993

WYBO, J.L., Introduction aux cyndiniques. Editions ESKA, 1998

\section{NOTES}

1. Le fameux logiciel surdimensionné et inadapté.

2. mobilisée notamment après les grandes catastrophes comme Bhopal en 1984 et Tchernobyl en 1986

3. pour : "Quality, Health, Safety, Environment »

4. Ce qui serait déjà une action de maîtrise du risque.

5. voir "des risques et des hommes", cassettes vidéos (Les films Roger Leenhardt, Eurisys Consultants) disséquant les causes et les séquences des accidents tes que TENERIFE, BHOPAL, PIPER ALPHA, etc. 


\section{RÉSUMÉS}

L'équipe projet constitue un microcosme particulièrement intéressant pour l'étude de la communication sur le risque dans l'entreprise. Au moyen de quelques exemples, les auteurs montrent que la prise de conscience du risque est une nécessité. Toutefois, ils montrent également que la communication sur le risque peut provoquer des effets néfastes à la bonne vie du projet si des mesures appropriées ne sont pas appliquées. Pour éviter une telle conjoncture, il faut dépasser la seule prise de conscience du risque par l'équipe pour arriver au stade de la mise en confiance des acteurs du projet. Cette transition doit se faire à l'aide d'outils concrets et symboliques, d'une part, et d'une organisation de l'équipe basée sur le principe de la vigilance mutuelle, d'autre part.

À project team seems to be an appropriate environment to study communication about risk in the enterprise. Using few examples, we want to show that taking conscientiously risk into account is a necessity. But it might be done with some precautions in order to avoid any bad effects that communication about risk could probably generate. Communication has to be done with the objective to develop a good degree of confidence for all project team members on their capacity to challenge. Such a transition is promoted by specific tools that are described, and by an organisation of the team based on mutual supervising.

INDEX

Mots-clés : communication, organisation par projets, maîtrise des risques

\section{AUTEURS}

\section{DIDIER GOURC}

D. Gourc : enseignant chercheur à l'École des Mines d'Albi, thèse en génie industriel au LISPA (Laboratoire d'Ingénierie des Systèmes de Production et d'Automatique) de l'École d'Ingénieurs de Tours sur la réingénierie des systèmes de production, soutenue en 1997.

\section{BÉATRICE VACHER}

B. Vacher : enseignant chercheur à l'École des Mines d'Albi, thèse en gestion au CRG de l'École Polytechnique sur « la gestion de l'information en entreprise », soutenue en 1996.

\section{HERVÉ PINGAUD}

H. Pingaud: Professeur au centre de recherche en Génie Industriel de l'École des Mines d'Albi, habilité à diriger les recherches de l'INP de Toulouse en 1997. 